\title{
Patients unaware of their HIV status present to an inner city accident and emergency department with respiratory complications
}

\author{
Rachel Landau, Richard Coker, Els Vermeulen, Robin Touquet, Jane Fothergill, \\ Mark C Poznansky
}

St Mary's Hospital, London W2 1NY, United Kingdom: Department of Accident and Emergency Medicine $R$ Landau $\mathrm{R}$ Touquet J Fothergill

Department of Respiratory Medicine R Coker

\section{Department of Genito-Urinary Medicine and Communicable Diseases E Vermeulen}

Harvard Medical School, Boston, MA 02115, USA:

Division of Infectious Diseases Medicine Mark C. Poznansky Correspondence to $\mathrm{Dr}$ Richard Coker.

Accepted for publication 27 March 1997

\begin{abstract}
Objective-To examine the clinical presentations and management of patients presenting to an accident and emergency (A\&E) department with an AIDS defining illness (ADI).

Methods-Presentations of patients in the A\&E department with ADI were reviewed retrospectively. The age, sex, ethnic origin, risk factor for HIV infection, route of referral to hospital, presenting complaint, triage category, referral from $A \& E$, admission under medical specialists, diagnosis, and survival from ADI were noted for each patient.

Results -133 patients were registered at $\mathrm{St}$ Mary's Hospital in London with ADI during 1994. A significant minority of these patients $(25 / 133)$ presented to the hospital without prior knowledge of their HIV positive status. Thirty two patients presented to the A\&E department with their ADI. Of these, 13/32 (41\%) were unaware of their HIV serostatus. All 13 patients had an acute respiratory disease (Pneumocystis carinii pneumonia or pulmonary tuberculosis). In contrast, patients aware of their HIV positive status (19/32) presented to the A\&E department with a wide range of non-pulmonary ADI.

Conclusions-The study emphasises the importance of respiratory complications in patients who present with an ADI to emergency departments but are unaware of their HIV positivity. These patients presented solely with Pneumocystis carinii pneumonia or pulmonary tuberculosis, conditions in which early diagnosis and treatment significantly reduce morbidity and mortality.

(F Accid Emerg Med 1997;14:304-306)
\end{abstract}

Keywords: AIDS defining illness; accident and emergency department; HIV status

Patients continue to present to the accident and emergency (A\&E) department at St Mary's Hospital with AIDS defining illnesses (ADI) while being unaware of their HIV serostatus. ${ }^{1}$ This occurs despite the provision of a well publicised confidential HIV testing and treatment centre at the hospital. Patients who present to the hospital in this way pose a considerable challenge with regard to the acute management of their ADI. ${ }^{1-5}$ It is clear from anonymous serosurveys in inner city hospitals including St Mary's that the seroprevalence of HIV among hospital subpopulations, such as $\mathrm{A} \& \mathrm{E}$ attenders, is considerably greater than the rate for the population of individuals which the hospital serves. For example, St Mary's Hospital consistently makes approximately one fifth of its annual new AIDS diagnoses in individuals who are unaware of their HIV status at the time of their ADI. ${ }^{6}$ The correct management and referral of patients with an ADI who are unaware of their HIV serostatus is particularly important in the A\&E department; it is in $A \& E$ that patients are referred to specialist medical teams. ${ }^{578-9}$ Respiratory complications are a recognised manifestation of HIV disease. ${ }^{10}$ Inappropriate referral or management may ultimately lead to inadequate treatment. ${ }^{5711}$

In this retrospective study we examined the clinical presentations and management of all patients presenting to the $\mathrm{A} \& \mathrm{E}$ department at St Mary's with an ADI, with and without knowledge of their HIV positive status, during 1994.

\section{Methods}

We performed a retrospective study on patients presenting to St Mary's Hospital with their ADI between the 1 January 1994 and 31 December $1994 .^{12}$ The patients were divided into two groups, consisting of patients who were unaware (group A) or aware (group B) of their HIV positive status at the time of their ADI. A single ADI which precipitated the diagnosis of AIDS was noted for each patient.

For each patient the age at diagnosis of first ADI, sex, ethnic origin, country of origin, risk factors for HIV-1 infection, route of referral to hospital, presenting complaint, triage category, referral from the A\&E department, admission under medical specialists, treatment, and diagnosis were noted, and the time between the HIV positive test and the ADI was determined. Haemoglobin concentration, neutrophil count, mean red cell volume (MCV), and $\mathrm{CD} 4$ lymphocyte count were also noted at the time of ADI.

\section{STATISTICAL METHODS}

All categorical variables were compared between the two groups using Fisher's exact test. The Mann-Whitney $U$ test was used for comparison of all other continuous variables. 
Table 1 Route of admission of patients with an AIDS defining illness (ADI) to St Mary's Hospital: 133 patients were admitted to the hospital with ADI during 1994; patients were referred to hospital through the accident and emergency $(A \mathcal{E} E)$ department, the walk in clinic for patients with HIVIAIDS, from their general practitioner, or from a medical outpatient department (medical OPD)

\begin{tabular}{lllll}
\hline & Through AEE & From HIV clinic & From GP & From medical OPD \\
\hline Group A ( $\mathrm{n}=25)$ & $13(52 \%)$ & $0(0 \%)$ & $6(24 \%)$ & $6(24 \%)$ \\
Group B $(\mathrm{n}=108)$ & $19(17 \%)$ & $71(66 \%)$ & $18(17 \%)$ & $0(0 \%)$ \\
P value & $<0.001$ & & & \\
\hline
\end{tabular}

^ Fisher's exact test.

\section{Results}

One hundred and thirty three patients presented to St Mary's Hospital with their ADI between the 1 January 1994 and 31 December 1994. The majority of patients in group A (patients unaware of their HIV serostatus on admission to hospital) presented with their $\mathrm{ADI}$ to the $\mathrm{A} \& \mathrm{E}$ department $(13 / 25,52 \%)$. The remaining patients in group $A$ were admitted to the hospital at the request of their general practitioner or through the medical outpatient department (table 1). In contrast, the majority of patients in group B (patients aware of their HIV seropositivity on admission) were admitted from the HIV clinic attached to the genito-urinary medicine department at the hospital. Only 19/108 patients in group B ( $17 \%$ ) were admitted through the $A \& E$ department. All patients entering A\&E with an ADI were triaged into a major illness category, defined as a patient potentially requiring urgent hospital admission. The A\&E department at St Mary's sees approximately 28000 patients a year aged between 16 and 50 years old and admits approximately 3100 of them.

All patients who were unaware of their HIV status (group A) who first attended the $A \& E$ department with their ADI presented with symptoms and signs of acute respiratory diseases which were shown after admission to be due to Pneumocystis carinii infection (9/13) or pulmonary tuberculosis (4/13) (table 2). In contrast, patients aware of their HIV status (group B) presented with a range of symptoms and signs from a variety of opportunistic infections (table 2). The three cases of tuberculosis that occurred in group B were of extrapulmonary origin.

The members of the groups A and B admitted through the A\&E department did not vary with respect to their age at presentation with their ADI, A\&E triage category, sex, ethnic origin, the ratio of United Kingdom citizens to non-UK citizens, or risk factors for HIV infection (table 3), or in their $\mathrm{Hb}$, neutrophil count, and CD4 positive T lymphocyte count (data not shown). All patients in group $\mathrm{A}$ attending $\mathrm{A} \& \mathrm{E}$ with an $\mathrm{ADI}$ were subsequently referred to the on call general medical team, whereas all the patients in group B, who were aware of their HIV positive status, were referred to the on call HIV medicine team. The proportion of patients who died from Pneumocystis carinii pneumonia within three months of admission through the $A \& E$ department was the same for both groups (group A, 1/13 deaths; group B, $2 / 19$ deaths).

\section{Discussion}

Between 1991 and 1994 approximately one fifth of patients presenting with an $\mathrm{ADI}$ attended St Mary's Hospital in London without prior knowledge of their HIV positive serostatus. ${ }^{1}$ During 1994, 25/133 patients (19\%) attended St Mary's Hospital in this category. Of these 25 patients, 13 attended the A\&E department as their first port of call in the hospital with symptoms of Pneumocystis carinii pneumonia or pulmonary tuberculosis. These patients pose a considerable challenge with regard to their acute management in the $A \& E$ department, their subsequent referral, and the management of their coincidental $\mathrm{ADI}$ and first positive HIV test. ${ }^{11}$

All the patients in group A presented to the A\&E department with acute respiratory illnesses which were diagnosed as either Pneumocystis carinii pneumonia or pulmonary tuberculosis. This was in contrast to patients in group $B$ who presented to the $A \& E$ department with a wide range of $\mathrm{ADI}$. The use of primary pneumocystis prophylaxis by patients aware of their HIV status and the higher prevalence of tuberculosis in regions from which the patients came who were unaware of their HIV status are the most likely explanations for the differing clinical pattern between the two groups at presentation to A\&E. These findings emphasise the importance of having a high index of suspicion of underlying HIV disease when admitting and treating young adult patients with respiratory infections in A\&E. A high index of suspicion for HIV related illness is further justified by a recently completed serosurvey of patients attending the A\&E department at St Mary's Hospital which showed that of 1503 patients attending the department during December 1994 and January 1995, one in 30 males and females $(2.4 \%)$ between the ages of 16 and 49 were HIV positive. ${ }^{6}$ Of these HIV positive

Table 2 AIDS defining illness (ADI) of patients asmitted to St Mary's Hospital and the dubgroup of patients admitted through accident and emergency $(A \mathcal{E} E)$. Number and percentages of individual $A D I$ are shown

\begin{tabular}{|c|c|c|c|c|c|c|c|c|c|c|c|}
\hline & $P C P$ & $K S$ & $O C$ & $C M W$ & $W S$ & $N H L$ & $M T B$ & Cryptsp. & Toxo & $M A I$ & Other \\
\hline \multicolumn{12}{|c|}{ ADI on presentation to St Mary's Hospital } \\
\hline Group A $(n=25)$ & $11(44)$ & $5(25)$ & $3(12)$ & $0(0)$ & $0(0)$ & $0(0)$ & $5(16)$ & $0(0)$ & $1(4)$ & $0(0)$ & $0(0)$ \\
\hline Group $B(n=108)$ & $30(28)$ & $14(12)$ & $13(13)$ & $12(11)$ & $4(3)$ & $3(2)$ & $9(9)$ & $7(8)$ & $4(4)$ & $3(3)$ & $9(10)$ \\
\hline$P$ value & $\mathrm{NS}^{\star}$ & & & & & & & & & & \\
\hline \multicolumn{12}{|c|}{ ADI on presentation to accident and emergency } \\
\hline Group A (n=13) & $9(69)$ & $0(0)$ & $0(0)$ & $0(0)$ & $0(0)$ & $0(0)$ & $4(31)$ & $0(0)$ & $0(0)$ & $0(0)$ & $0(0)$ \\
\hline Group B $(n=19)$ & $3(16)$ & $0(0)$ & $1(5)$ & $1(5)$ & $0(0)$ & $0(0)$ & $3(16)$ & $4(21)$ & $4(21)$ & $0(0)$ & $3(16)$ \\
\hline$P$ value & $<0.01$ & & & & & & & & & & \\
\hline
\end{tabular}

^ Fisher's exact test.

PCP, Pneumocystis carinii pneumonia; KS, Kaposi's sarcoma; OC, oesophageal candidiasis; CMV, cytomegalovirus infection; WS, wasting syndrome; NHL, non-Hodgkin's lymphoma; MTB, $M$ tuberculosis infection; Cryptsp, cryptosporidiosis; Toxo, toxoplasmosis; MAI, Mycobacterium avium intracellulare; Others include Samonella typhi septicaemia. 


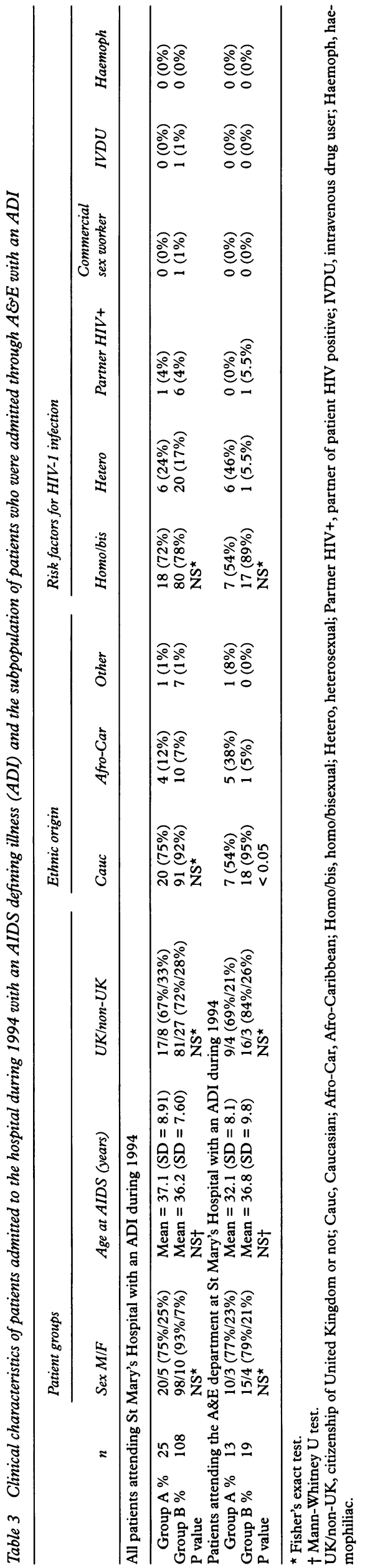

patients in the anonymous serosurvey, only one quarter had declared their HIV positive status to health care workers in the $A \& E$ department during their admission. ${ }^{6}$

Our study shows that the group of patients who attend the $A \& E$ department without prior knowledge of their HIV positive status do not differ significantly with respect to age, sex ratio, or country of origin from those who know that they are HIV positive. There was an excess of Afro-Caribbean and heterosexual patients in the group unaware of their HIV status, but this did not reach statistical significance. Previous studies of patients presenting to inner London hospitals have shown a preponderance of heterosexual Afro-Caribbean patients in the group of patients who present with an ADI without knowing that they are HIV positive. ${ }^{13}$ In addition, the fact that their short term survival from their ADI did not differ between the two groups may reflect the high level of experience in treating these conditions which currently exists at St Mary's Hospital. ${ }^{4}$

In conclusion, this study emphasises the important role of $A \& E$ and respiratory medicine clinicians in the management of the group of young adult patients who present to an inner city hospital through the A\&E department with an ADI such as Pneumocystis carinii pneumonia or pulmonary tuberculosis without knowing about their HIV positive status. A high index of suspicion for these diseases in inner city $A \& E$ departments will result in improved clinical management of these patients.

1 Poznansky MC, Coker R, Skinner C, Bailey S, Hill A, Whitaker L, et al. HIV positive patients first presenting with an AIDS defining illness: characteristics and survival. BM 1995;311:156-8

2 Drotman DP. Earlier diagnosis of human immunodeficiency virus (HIV) infection and more counseling. Ann Intern virus (H) 1989;110:680-1.

3 Porter K, Wall G, Evans B. Factors associated with the lack of awareness of $\mathrm{HIV}$ infection before diagnosis of AIDS. of awareness of HIV

4 Peters BS, Beck EJ, Coleman DJ, Wadsworth MJH, McGuiness O, Harris JRW, et al. Changing disease patterns in patients with AIDS in a referral centre in the United Kingdom: the changing face of AIDS. BMJ 1991;302 203-7.

5 Coker RJ, Desmond N, Poznansky MC, Smith C, Bell D, Riordan JF, et al. Experience of HIV disease in a London district general hospital. Int J STD AIDS 1995;5:47-9.

6 Poznansky MC, Walters J, Cruikshank A, Pollock $R$ Dendrowskyj $\mathrm{P}$, Lewis $\mathrm{K}$, et al. The rising prevalence of HIV-1 infection in patients attending an inner city acciden and emergency department. J Accid Emerg Med 1996;13. 424-5.

7 Coker RJ, Nieman R, McBride M, Mitchell DM, Harris JRW, Weber JN. Co-trimoxazole versus dapsonepyrimethamine for prevention of Pneumocystis carinii pyrimethamine for prevention of

8 Kitata MM, Koepsell TD, Deyo RA, Maxwell CL, Dodge WT, Wagner EH. Physician's experience with the acquired immunodeficiency syndrome as a factor in patients' immunodeficiency syndrome as a facto

9 Coker RJ, Poznansky MC. Physicians' experience and Coker RJ, Poznansky MC. Physicians' experience and
survival in patients with AIDS [letter]. N Engl J Med 1996; 335:349.

10 Miller R. HIV-associated respiratory diseases. Lancet 1996; 348:307-12.

11 Asimos AW, Ehrhardt J. Radiographic presentation of pulmonary tuberculosis in severely immunosuppressed HIV-seropositive patients. Am J Emerg Med 1996;14:35963.

12 Centers for Disease Control. Revision of the CDC surveillance definition for acquired immunodeficiency syndrome. MMWR 1987;36(suppl):3-15s.

13 Petruckevitch A, Del Amo J, Phillips A, Stephenson J, Johnson A, DeCock KM. Survival and CD4 lymphocyte declin in HIV-infected Africans and non Africans in London [abstr]. Tenth International AIDS Conference, Vancouver, 1996:abstract B3337. 